\title{
Evaluation of an Algorithm for the Recognition and Classification of Proper Names
}

\author{
Takahiro Wakao Robert Gaizauskas Yorick Wilks \\ Department of Computer Science, \\ University of Sheffield \\ \{T.Wakao, R.Gaizauskas, Y.Wilks\}@dcs.shef .ac.uk
}

\begin{abstract}
We describe an information extraction system in which four classes of naming expressions - organisation, person, location and time names - are recognised and classified with nearly $92 \%$ combined precision and recall. The system applies a mixture of techniques to perform this task and these are described in detail. We have quantitatively evaluated the system against a blind test set of Wall Street Journal business articles and report results not only for the system as a whole, but for cach component technique and for each class of name. These results show that in order to have high recall, the system needs to make use not only of information internal to the naming expression but also information from outside the name. They also show that the contribution of each system component varies from one class of name expression to another.
\end{abstract}

\section{Introduction}

The appropriate treatment of proper names is essential in a natural language understanding system which processes unedited newswire text, since up to $10 \%$ of this type of text may consist of proper names (Coates-Stephens, 1992). Nor is it only the sheer volume of names that makes them important; for some applications, such as information extraction (IE), robust handling of proper names is a prerequisite for successfully performing other tasks such as template filling where corroctly identifying the entities which play semantic roles in relational frames is crucial. Recent research in the fifth and sixth Message Understanding Conferences (MUC5, 1993) (MUC6, 1995) has shown that the recognition and classification of proper names in business newswire text can now be done on a large scale and with high accuracy: the success rates of the best systems now approach $96 \%$.

We have developed an IE system - LaSIE (Large Scale Information Extraction) (Gaizauskas et al, 1995) - which extracts important facts from business newswire texts. As a key part of the extraction task, the system recognises and classifies certain types of naming expressions, namely those specified in the MUC-6 named entity (NE) task definition (MUC6, 1995). These include organisation, person, and location names, time expressions, percentage expressions, and monetary amount expressions. As defined for MUC-6, the first three of these are proper names, the fourth contains some expressions that would be classified as proper names by linguists and some that would not, while the last two would generally not be thought of as proper names. In this paper we concentrate only the behaviour of the LaSIE system with regards to recognising and classifying expressions in the first four classes, i.e. those which consist entirely or in part of proper names (though nothing hangs on omitting the others). The version of the system reported here achieves almost $92 \%$ combined precision and recall scores on this task against blind test data.

Of course the four name classes mentioned are not the only classes of proper names. Brand names, book and movie names, and ship names are just a few further classes one might chose to identify. One might also want to introduce subclasses within the selected classes. We have not done so here for two reasons. First, and foremost, in order to generate quantitative evaluation results we have used the MUC-6 datid and scoring resources and these restrict us to the above proper name classes. Secondly, these four name classes account for the bulk of proper name occurrences in business newswire text. Our approach could straightforwardly be extended to account for additional classes of proper names, and the points we wish to make about the approach can be adequately presented using only this restricted set.

Our approach to proper name recognition is heterogeneous. We take advantage of graphological, syntactic, semantic, world knowledge, and discourse level information to perform the task. In the paper we present details of the approach, describing those data and processing components of the overall IE system which contribute to proper name recognition and classification. Since name 
recognition and classification is achicved through the activity of four successive components in the system, we quantitatively evaluate the successive contribution of each component in our overall approach. We perform this analysis not only for all classes of names, but for each class separately. The resulting analysis

1. supports McDonald's obscrvation (McDonald, 1993) that external evirlence as well as internal evidence is esscntial for achieving high precision and recall in the recognition and classification task; i.e. not just the name string itself must be examined, but other information in the text must be used as well;

2. shows that all components in our hoterogencous approach contribute significantly;

3. shows that not all classes of proper names benefit equally from the contributions of the different, components in our system: in particular, organisation names bencfit most from the use of external evidence.

In the second section an overview of the IaSIE system is presented. The third scction explains in detail how proper names are recognised and classified in the system. The results of cvaluating the system on a blind test set of 30 articles are presented and discussed in section 4 . Section 5 concludes the paper.

\section{LaSIE system overview}

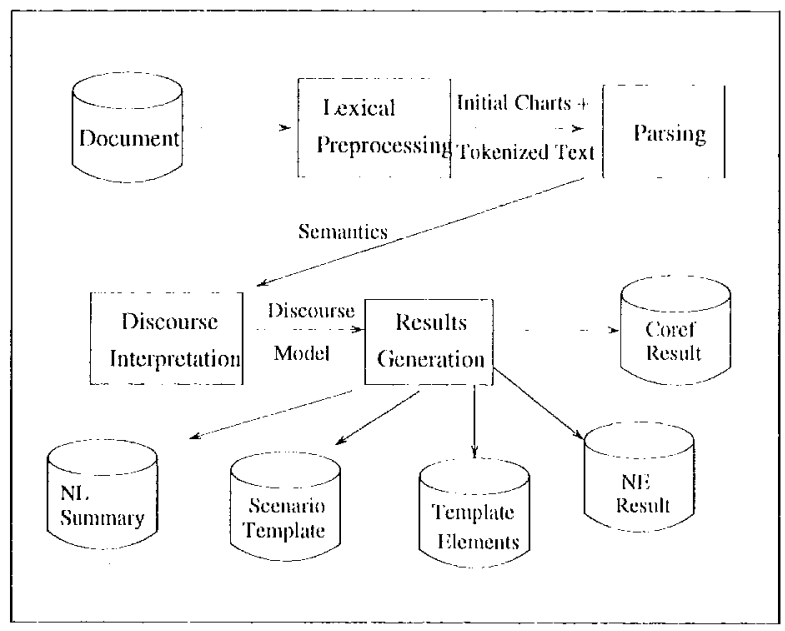

Figure 1: LaSIE System Architecture

LaSII has been designed as a general purpose IE rescarch system, initially geared towards, but not, solely restricted to, carrying out the tasks specified by the sixth Message Understanding Conference: named entity recognition, coreference resolution, template element filling, and scenario template filling tasks (see (MUC6, 1995) for further details of the task descriptions). In addition, the system can generate a brief natural language summary of the scenario it. has detected in the text.
All of these tasks are carried out by building a single rich model of the text - - the discourse model from which the various results are read off.

The high level structure of IadSIE is illustrated in Figure 1. 'The system is a pipelined architecture which processes a text sentence-ati-a-time and consists of three principal processing stages: lexical preprocessing, parsing plus semantic interpretation, and discourse interpretation. The overall contributions of these stages may be briefly described as follows:

- lexical preprocessing reads and tokenises the raw input text, tags the tokens with parts-of-speech, performs morphological andalysis, performs phrasal matching against lists of proper names, and builds lexical and phrasal chart edges in a feature-based formalism for hand-over to the parser;

- parsing does two pass parsing, pass one with a special proper name grammar, pass two with a general grammar and, after sclecting a 'best parse', passes on a semantic representation of the current sentence which inclucles name class information;

- discourse interpretation adds the inform ation in its input semantic representiation to a hierarchically structured semantic; net, which encodes the system's world model, adds additional information presupposed by the input to the world model, performs coreference resolution between new instances adcled and others already in the world model, and adds information consequent upon the addition of the input to the world model.

For further details of the system see (Gaiyduskas et al, 1995)

\section{How proper names are recognised and classified}

As indicated in section 1, our approach is a hetcrogeneous one in which the system makes use of graphological, syntactic, somantic, world knowledge, and discourse level information for the rocognition and classification of proper names. The system utilises both the information which comesi from the name itself (internal evidence in McDonald's sense (McDonald, 1993)) as well as the information which comes from outside the name, from its context in the text (external evidence).

In what follows wo describe how proper names are recognised and classified in LaSIE by considering the contribution of each system component.

\subsection{Lexical preprocessing}

The input text is first tokenised and then each token is tagged with a part-of-speech tag from the Penn 'Treebank tagset (Marcus et al, 1993) using a slightily customised ${ }^{1}$ version of Brill's tag-

${ }^{1}$ The tagger has been customised by adding some entries to its lexicon and by adding several special tags 
ger (Brill, 1994). The tagset contains two tags for proper nouns ... NNP for singular proper nouns and NNPS for plurals. The tagger tags a word as a proper noun as follows: if the word is found in the tagger's lexicon and listed as a proper noun then tag it as such; otherwise, if the word is not found in the lexicon and is uppercase initial then tag it as a proper noun. Thus, capitalised unknown tokens are tagged as proper nouns by default.

Before parsing an attempt is made to identify proper name phrases - sequences of proper names and to classify them. This is done by matiching the input against pre-stored lists of proper names. These lists are compiled via a flex program into a finite state recogniser. Wach sentence is fed to the recogniser and all single and multi-word matches are tagged with special tags which indicate the name class.

Lists of names used include:

- organisation : about 2600 company and governmental institution names based on an organisation name list which was semiautomatically collected from the MUC-5 answer keys and training corpus (Wall Street Journal articles);

- location : about 2200 major country, province/state, and city names derived from a gazetteer list of about 150,000 place names;

- person : about 500 given names taken from a list of given names in the Oxford Advanced Lcarner's Dictionary (Hornby, 1980);

- company designator : 94 designators (e.g. 'Co.',PLC'), based on the company designator list provided in the MUC6 reference resources.

- human titles : about 160 titles, (c.g. 'President,',Mr.'), manually collected;

As well as name phrase matching, another technique is applied at this point. Inside multi-word proper names, certain words may function as trigger words. A trigger word indicates that the tokens surrounding it are probably a proper name and may reliably permit the class or cven subclass $^{2}$ of the proper name to be determined. For example, 'Wing and Prayer Airlines' is almost certainly a company, given the presence of the word 'Airlines'. Trigger words are detected by matching against lists of such words and are then specially tagged. Subsequently these tags are used by the proper name parser to build complex proper name constituents.

The lists of trigger words are:

- Airline company: 3 trigger words for finding airline company names, e.g. 'Airlines';

- Governmental institutions: 7 trigger words for governmental institutions, e.g 'Ministry';

for word classes such as days of the weck and months.

${ }^{2}$ company and governmental institution are subclasses of the class organisation. airline is a subclass of company.
- Location: 8 trigger words for location names, e.g. 'Gulf';

- Organisation: 135 trigger words for organisation names, e.g 'Association'.

These lists of trigger words were produced by hand, though the organisation trigger word lists werc generated semi-automatically by looking at organisation names in the MUC-6 training texts and applying certain heuristics. So, for example, words were collected which come immediatcly before 'of' in those organisation names which contain 'of', e.g. 'Association' in 'Association of Air Flight Attendants'; the last, words of organisation names which do not contain 'of' were examined to find trigger words like 'International'.

\subsection{Grammar rules for proper names}

The LaSIE parser is a simple bottom-up chart parser implemented in l'rolog. The grammars it processes are unification-style foature-based context frec grammars. During parsing, semantic rep resentations of constituents are constructed using Prolog term unification. When parsing ceases, i.e. when the parser can generate no further odges, a 'best parso selection' algorithm is run on the final chart, to chose a single analysis. The semantics are then extracted from this analysis and passed on to the discourse interpreter.

Parsing takes place in two passes, cach using a separate grammar. In the first pass a special grammar is used to identify proper names. These constituents are then treated as unanalysable units during the second pass which employs a more gencral 'sentence' grammar.

Proper Name Grammar The grammar rules for proper names constitute a subset of the system's noun phrase (NP) rules. All the rules wore produced by hand. There are 177 such rules in total of which 94 are for organisation, 54 for person, 11 for location, and 18 for time expressions.

Here are some examples of the proper name grammar rulos:

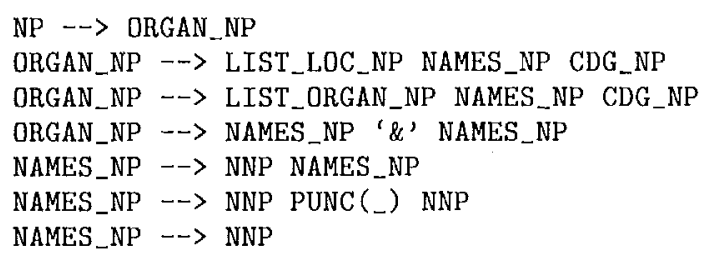

The non-terminals LIST_LOC_NP, LIST_ORGAN_NP and CDGNP are lags assigned to one or more input tokens in the name phrase tagging stage of lexical preprocessing. 'The non-terminal NNP is the tag for proper name assigned to a single token by the Brill tagger.

The rule ORGAN_NP $\rightarrow->$ NAMES_NP ' $\&$ ' NAMES_NP means that if an as yet unclassified or ambiguous proper name (NAMES_NP) is followed by ' $\&$ ' and another ambiguous proper name, then it is an organisation name. So, for example, 'Marks \& Spen- 
cer' and 'American 'Tolephono \& 'T'elegraph' will be classified as organisation names by this rule.

Nearly half of the proper name rules aro for organisation names because they may contain further proper names (e.g. person or location mames) as well as normal nouns, and their combinations. 'There are also a good number of rules for person names since care must be taken with given names, family names, titiles (c.g. 'Mr.',President'), and special lexical items such as 'de' (as in 'J. Ignacio Lopez de Arriortua') and 'Jr.', 'T', etc.

There are fewer rules for location names, as they are identified mainly in the previous preprocessing stage by look-up in the mini-gazettees.

Sentence Grammar Rules T'he grammar used for parsing at the sentence level contiains approximately 110 rules and was derived antomatically from the Penn TreeBank-II (P'TB-II) (Marcus et al, 1993), (Marcus et al, 1995). When parsing for a sentence is complete the resultant chart is analysed to identify the 'best parse'. From the best parse the associated semantices are extracted to be passed on to the disconrse interpreter.

Rules for compositionally constructing somantic representations were assigned by hanel to the grammar rules. For simple verbs and nouns the morphological root is used as a predicate name in the semantics, and tense and number features are translated directly into the semantic representation where appropriate. For named entities a token of the most specific type possible (c.g. company or perhaps only object) is created and a name attribute is associated with the entity, the attribute's value being the surface string form of the name. So, for example, assuming 'Ford Motor Co.' has already been classified as a company name, its semantic representation will be something liko company (e23) \& name (e23, 'Ford Motor Co.').

\subsection{Discourse interpretation}

The discourse interpreter module porforms two activitics that contribute to proper name classification (no further recognition of proper narnes goes on at this point, only a refining of their classification). The first activity is coreference resolution an unclassified name may be coreferred with a previously classified one by virtue of which the elass of the molassified name becomes known. The second activity, which is arguably not properly 'cliscourse interpretation' but nevertheless takes place in this module, is to perform inferences about the semantic types of argmments in certain relations; for example, in compound nominals such as 'Lrikson stocks' our somantic interpreter will tell us that there is a qualifier relation between 'Erikson' and 'stocks' and since the system stores the fact that named entities qualifying things of type stock are of type company it can classify the proper name 'Trikson' as a company.
Note that both of these techniques make use of external evidence, i.e. roly on information sup)plied by the context beyond the words in the instance of the proper name being classified.

\subsubsection{Proper name coreference}

Coreference resolution for proper namos is carried out in order to recognise altemative forms, especially of organisation names. For example, 'Ford Motor Co.' might be used in a text when the comprany is first mentioned, but subsequent: references are likely to be to 'Gord'. Similarly, 'Creative Artists Agency' might be abbreviated to 'CA ' later on in the same bext. Such shortened forms must be resolved as names of the samo organisation.

In order to determine whether given two proper names match, various heuristies are used. For example, given two names, Named and Name2:

- if Name2 is consists of an initial subscenuence of the words in Namel then Name2 matches Namel e.g. 'American Airlines Co.' aud 'Anerican Airlines';

- if Namel is a person name and Name2 is either the first, the family, or both names of Namel, then Name2 matches Namel e.g. 'John J. Maljor Jr.' and 'John Major'.

There are 31 such houristic rules for matching organisation nannes, 11 hencistics for person namres, and 3 rules for location namess.

Whon an unclassified proper noun is matched with a previously classified proper name in the text, it is marked as a proper name of the class of the known proper name. 'Thus, when we know 'Ford Motor Co.' is an organisation name but have not classified 'Ford' in the sanne text, coreference resolution determines 'Ford' to be an organisation name

\subsubsection{Semantic: Type Inference.}

In the following contexts, semantic type information about the types of arguments in certain relations is used to drive inferences permitting the classification of proper names. The system luses these tochniques in a fairly limited and experimontal way at present, and there is much room for their extension.

- noun-noun qualification: when an unclassi. fied proper name qualifies an organisationrelated thing then the name is classified as in organisation; o.g. in 'Frickson stocks' since 'stock' is semantically typed as an organisation-related thitng, 'Frickson' gots classificel ass an organisation name.

- possessives: when an unclassified proper nane stands in a possessive relation to an organisation post, then the name is classified as an organisation; e.g. 'vice president of $\triangle \mathrm{BC}$ ', 'ABC's vico president'.

- apposition : when an unclassilied proper name is apposed with a known location name, 
the former name is also classified as a location; e.g. given 'Fort Lauderdale, Fla.' if we know 'Fla.' is a location name, 'Fort Lauderdale' is also classified as a location name.

- verbal arguments: when an unclassified proper name names an entity playing a role in a verbal frame where the semantic type of the argument position is known, then the name is classified accordingly; e.g. in 'Smith retired from his position as ...' we can infor that 'Smith' is a person name since the semantic type of the logical subject of 'retire' (in this sense) is person.

\section{Results and Evaluation}

After these processing stages, the results generator produces a version of the original text in which all the proper names which have been detected are marked up with pre-defined SGML tags, spocifying their classes. These marked up texts are then automatically scored against manually marked up texts.

A series of evaluations has been donc on the system using a blind test set consisting of $30 \mathrm{Wall}$ Street Journal texts. In these texts there are 449 organisation names, 373 person names, and 110 location names and 111 time expressions in total. The overall precision and recall scores for the four classes of proper names are shown in Table 1.

\begin{tabular}{|l|c|c|}
\hline Proper Name Class & Recall & Precision \\
\hline Organisation & $91 \%$ & $91 \%$ \\
\hline Person & $90 \%$ & $95 \%$ \\
\hline Location & $88 \%$ & $89 \%$ \\
\hline Time & $94 \%$ & $97 \%$ \\
\hline Overall & $91 \%$ & $93 \%$ \\
\hline
\end{tabular}

Table 1: Overall Precision and Recall Scores

\subsection{System module contribution}

We have analysed the results in terms of how much each module of the system contributes to the proper name task.

Table 2 illustrates the contribution of each system module to the task for all classes of proper names. In addition to recall and precision scores, we have added Van Rijsbergen's F-measure which combines these scores into a single measure (Rijsbergen, 1979). The F-measure (also called P\&R) allows the differential weighting of precision and recall. With precision and recall weighted equally it is computed by the formula:

$$
F=\frac{2 \times \text { Precision } \times \text { Recall }}{\text { Precision }+ \text { Recall }}
$$

There are four different settings of the system.

- setting 1 : Only the lexical preprocessing techniques are used - part-of-speech tagging and name phrase matching.
- setting 2 : Two-stage parsing is added to 1 .

- setting 3 : Coreference resolution for proper names is added to 2 .

- setting 4 : Full discourse interpretation is added to $\mathbf{3}$. This is the full-fledged system

\begin{tabular}{|l|c|c|c|}
\hline Setting & Recall & Precision & P\&R \\
\hline 1 & 49 & 89 & 63.01 \\
\hline 2 & 79 & 94 & 85.82 \\
\hline 3 & 89 & 94 & 91.13 \\
\hline 4 & 91 & 93 & 91.75 \\
\hline
\end{tabular}

Table 2: Module Contribution Scores

Table 2 shows that we can attain reasonable results using tagging, exact phrase matching, trigger word detection, and parsing (setting 2). Note that this amounts to making use of only internal evidence. However, to achieve higher recall, we need coreference resolution for proper names (setting 3) and other context information (setting 4).

\subsection{Different classes of proper names}

We have also examined how the contribution of each component varies from one class of proper name to another.

For organisation names, using the same settings as above, scores are shown in Table $\mathbf{3}$.

\begin{tabular}{|l|c|c|c|}
\hline Setting & Recall & Precision & P\$सR \\
\hline 1 & 46 & 87 & 59.91 \\
\hline 2 & 65 & 92 & 76.15 \\
\hline 3 & 87 & 93 & 89.84 \\
\hline 4 & 91 & 91 & 91.13 \\
\hline
\end{tabular}

Table 3: Module Contributions for Org Names

For person names, location names and time exprossions the results are shown in Tables 4-6.

\begin{tabular}{|l|c|c|c|}
\hline Setting & Recall & Precision & P\&R \\
\hline 1 & 47 & 88 & 61.64 \\
\hline 2 & 89 & 95 & 92.34 \\
\hline 3 & 90 & 95 & 92.14 \\
\hline 4 & 90 & 95 & 92.14 \\
\hline
\end{tabular}

Table 4: Module Contributions for Person Names

Figure 2 shows graphically how the system components contribute for each of the four different classes of proper names as well as for all classes combincd.

\section{Conclusion}

We have described an IE system in which four classes of naming expressions (organisation, person, and location names and time expressions) are recognised and classified. The system was tested on 30 unseen Wall Street Journal texts and the results were analysed in terms of major systom components and different classes of referring expression. 


\begin{tabular}{|c|c|c|c|}
\hline Setting & Recall & Precision & T\&l \\
\hline 1 & 81 & 94 & 86.84 \\
\hline 2 & 88 & $-\frac{90}{98}$ & 88.99 \\
\hline 3 & 88 & $-\frac{89}{89}$ & 88.58 \\
\hline 4 & & 88.58 \\
\hline
\end{tabular}

Table 5: Module Contributions for Iocation Names

\begin{tabular}{|c|c|c|c|}
\hline Setting & Recall & Procision & P\&R \\
\hline 1 & 32 & 100 & 48.97 \\
\hline 2 & 94 & $97^{-}$ & $95 . \overline{1} 1$ \\
\hline 3 & 94 & 97 & 95.41 \\
\hline 4 & 94 & 97 & 95.41 \\
\hline
\end{tabular}

Table 6: Module Contributions for 'Time Expressions

Tables 3-6 and Figure 2 cnable us to make the following observations:

1. Techniques relying on internal evidence only exact word and phrase matching, graphological conventions, and parsing - are not sufficient to recognise and classify organisation names. It is clear that in order to have high recall for organisation names, we need to be able to make good use of extermal evidence as well, i.e. proper name coreference resolution and information from the surrounding context.

2. On the other hand, for person and location names and time expressions, techniques relying solely on intemal evidence do permit us to abtain high recall whilst maintaining high precision. Thus, the contribution of different, system components varies from one class of proper name to another.

3. However, given that in a reasonable sample of business newswire text, $43 \%$ of the proper names are organisation names, it is evident that for a system to achieve high overall precision and recall in the namo recognition and classification task on this text type, it must, utilise not only internal evidence but also external evidence.

More generally, two conclusions can be drawn. First, the results presented above suggest that, when a system for proper name recognition and/or classification is evaluated, much benefit can be gained by analysing it not only in terms of overall recall and precision figures, but, also in terms of system components and classes of names. Second, a heterogeneous approach to the recognition and classification of proper names in newswire text such as described hore is appropriate since it provides mechanisms that can utilise the variety of internal and external evidence which is available and which needs to be taken into account.

\section{Acknowledgements}

This rescarch has been made possible by the grauts from the U.K. Department of Trade and

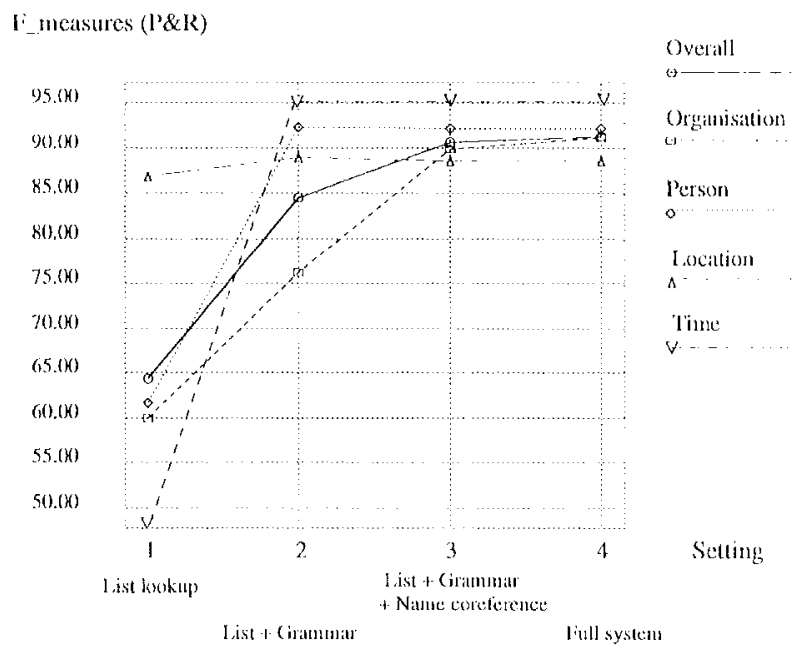

Figure 2: Contribution of System Components

Industry (Grant Red. YAJ/8/5/1002) and the Engineering and Physical Scienco Rescarch Conncil (Grant \# (GR/K25267).

\section{References}

Brill, Js. (1994). "Some Advances in Transformation Based Part of Specch Tagging." In I'roc. $A A A I$.

Coates-Stephens, S. (1992). The Aralysis and Acruzisition of I'roper Names for Robust Text Understand. ingl. PhI thesis, Department of Computer Scionese, City Univorsity, London.

Gaizauskas, R.; Wakao, 'T.; Humphreys, K.; Cumningham, H. and Wilks, Y. (1995). "University of Sheflicld: Description of JaSTE system as used for MUC-6." In Proccedings of the Sixth Message Understanding Conferenee (MUC-6), Morgan Kaufnana.

Hornby A.S. (Wd.). (1980). Oaford Advanced Learner's Dictionary of Current linglish. london: ()xford University Press.

Marcus, M.; Santorini, B, and Marcinkiewicz, M.A. (1993). "Building a Large Anuotated Conpus of English: The 'enm Theobank." Computational lin guistics, $19(2)$ : 313330 .

Marcus, M.; Kim, (1; Marcinkiewič, M.A.; MacIntyre, R.; Bies, $\Lambda$; Verguson, M.; Katz, K. and Schasherger, B. (1995). "The Penn Theobauk: Annotating P'redicate Argument Structure." Distributed on The Penn Treebank Release 2 CD-ROM by the Linguistic I)ata Consortiurn.

Mcloonald, D.1). (1993). "Internal and lixternal Evidence in the Irlentification and Semantic: Categorisation of Proper Names." In Procecdings of SIGLLIX workshop on "Acquisition of letrical Knowledge from Texl", pp. 3243.

MUC-5. (1993). Procecdings of the l'ifth Message Understanding Conference (MUC-5). Morgan Kautnnan.

MUC-6. (1995). Procecdings of the Sixth Message Un. derstanding Confenence (MUC-6). Morgan Kaufinan.

Vats Rijsbergen, (..J. (1979). Information Retricual London: Butterworths. 